\title{
FACTORES BIÓTICOS, ABIÓTICOS Y AGRONÓMICOS QUE AFECTAN LAS POBLACIONES DE ADULTOS DE MOSCA PINTA (HEMIPTERA: CERCOPIDAE) EN CULTIVOS DE CAÑA DE AZÚCAR EN VERACRUZ, MÉXICO
}

\author{
BIOTIC, ABIOTIC AND AGRONOMIC FACTORS THAT AFFECT ADULT \\ POPULATIONS OF SPITTLEBUG (HEMIPTERA: CERCOPIDAE) IN SUGAR \\ CANE CROPS IN VERACRUZ, MÉXICO
}

\author{
Juan Carlos GARCÍA-GONZÁLEZ, ${ }^{1}$ José LÓPEZ-COLLADO, ${ }^{1, *}$ CARLOS GiLberto \\ GARCÍA-GARCÍA, ${ }^{2}$ JuAn A. VILLANUEVA-JIMÉNEZ ${ }^{1}$ y MARTHA ElENA NAVA-TABLADA ${ }^{3}$
}

\author{
${ }^{1}$ Colegio de Postgraduados, Campus Veracruz. Carretera federal Veracruz-Xalapa km 88.5, Código Postal 91690, \\ Veracruz, México. <jlopez@colpos.mx>. \\ ${ }^{2}$ Colegio de Postgraduados, Campus Córdoba. Carretera federal Córdoba-Veracruz km 348, Congregación \\ Manuel León, Córdoba, Veracruz, Código Postal 94946. Veracruz, México. \\ ${ }^{3}$ El Colegio de Veracruz, Carrillo Puerto No. 26, Zona Centro, Código Postal 91000. Veracruz, México. \\ * Autor de correspondencia: <jlopez@colpos.mx>.
}

Recibido: 06/07/2017; aceptado: 11/09/2017.

Editor responsable: Magdalena Cruz.

García-González, J. C., López-Collado, J., García-García, C. G., Villanueva-Jiménez, J. A. y Nava-Tablada, M. E. (2017) Factores bióticos, abióticos y agronómicos que afectan las poblaciones de adultos de mosca pinta (Hemiptera: Cercopidae) en cultivos de caña de azúcar en Veracruz, México. Acta Zoológica Mexicana (n.s), 33(3), 508-517.

RESUMEN. Altas poblaciones de mosca pinta (Hemiptera: Cercopidae) pueden reducir el rendimiento de la caña de azúcar. El objetivo de este trabajo fue cuantificar la influencia de algunos factores bióticos, abióticos y agronómicos sobre las poblaciones de adultos de mosca pinta. Se compilaron registros del número de adultos capturados por trampa, grado de infestación previa de las parcelas, número de ninfas observadas, cobertura de maleza en el interior y en el borde de las parcelas, tiempo de muestreo, aplicación de insecticidas y entomopatógenos, precipitación y temperatura. Los muestreos se realizaron en 15 parcelas del 2 de julio al 15 de octubre de 2014 en Veracruz, México. Los datos se analizaron con un modelo lineal mixto. La mayoría de los ejemplares adultos capturados (98\%) fueron de Aeneolamia contigua (Walker, 1851). Los análisis indicaron los siguientes efectos significativos: se observó una relación positiva de las poblaciones de adultos con las ninfas de mosca pinta y la infestación del año previo de la parcela. Se presentó una relación directa entre la cobertura de malezas en el interior de la parcela y las poblaciones de adultos de mosca pinta. La relación de la maleza en el borde de la parcela con los adultos fue irregular y no significativa. Las poblaciones de ninfas tuvieron el efecto más claro sobre la presencia de poblaciones de adultos. La temperatura tuvo un efecto positivo significativo sobre los adultos, no así la precipitación ni el tiempo de muestreo. Se discuten los resultados en relación con aspectos de manejo preventivo de la mosca pinta.

Palabras clave: caña de azúcar, Aeneolamia spp., Prosapia sp., modelo lineal mixto.
García-González, J. C., López-Collado, J., García-García, C. G., Villanueva-Jiménez, J. A., \& Nava-Tablada, M. E. (2017) Biotic, abiotic and agronomic factors that affect adult populations of spittlebug (Hemiptera: Cercopidae) in sugar cane crops in Veracruz, Mexico. Acta Zoológica Mexicana (n.s), 33(3), 508-517.

ABSTRACT. High populations of the spittlebug (Hemiptera: Cercopidae) may reduce yields on sugar cane. This research was aimed to quantify the effect of biotic, abiotic and agronomic factors on adult populations of the spittlebugs. Data collected was related to the number of adults captured in traps, previous-year infestation level of the sampling plot, nymph counts, weed coverage within and on the border of the plots, sampling time, insecticide and entomopathogen applications, precipitation and temperature. Fifteen plots were sampled from July 2 to October 15, 2014 in Veracruz, Mexico. The data was analyzed as a linear mixed model. Most of the captured adults (98\%) were Aeneolamia contigua (Walker, 1851). The analysis showed the following significant effects: a positive relationship between adult populations and spittlebug nymphs and previous-year infestation, and a direct relationship between within-plot weed coverage and spittlebug adult populations. Weed coverage on the borders showed fluctuating effects and had not significant effect on adult populations. Temperature had a positive, significant effect as well, but precipitation and sampling time did not affect adult populations. Results are discussed as related to preventive spittlebug management.

Key words: Sugarcane, Aeneolamia spp., Prosapia sp., linear mixed model. 


\section{INTRODUCCIÓN}

El cultivo de la caña de azúcar es uno de los más importantes en México, genera más de dos millones de empleos en forma directa o indirecta en 230 municipios de 15 estados, con un valor aproximado de la producción de 30 mil millones de pesos (SIAP, 2017). El estado de Veracruz ocupa el primer lugar en superficie sembrada con 287 mil hectáreas, lo que representa $48 \%$ del total nacional (SIAP, 2017), y es el principal productor de caña de azúcar con aproximadamente 20 millones de toneladas al año (INEGI, 2014). Este cultivo presenta factores que limitan su producción en campo, como las plagas de insectos que generan pérdidas económicas; destacan la mosca pinta (Aeneolamia spp. y Prosapia spp.), los roedores y los barrenadores del tallo (Salgado et al., 2003). Las moscas pintas son originarias del continente americano y tienen una relación coevolutiva con los pastos. Al introducirse el cultivo de la caña de azúcar a México, estos insectos la tomaron como una planta hospedera, siendo hoy en día su principal plaga en áreas tropicales y subtropicales (Peck, 2001). Su distribución geográfica se concentra en el Golfo de México y en menor grado en el océano Pacífico (Flores, 1994). Los daños que causa este insecto plaga pueden ser cuantiosos y se estiman principalmente en las pérdidas en rendimiento, que oscilan entre 5 y 20 t ha $^{-1}$ (De la Cruz et al., 2005; García-García et al., 2006).

Se han realizado diferentes estudios relacionados con los factores que influyen en la incidencia de la mosca pinta en la caña de azúcar, pero usualmente se estudian los factores por separado. Diferentes autores mencionan que la precipitación y la temperatura son favorables para el desarrollo de la mosca pinta (Salazar \& Badilla, 1997; García-García et al., 2006). Respecto a la dinámica poblacional de adultos y ninfas, Evans (1972) y Badii \& Castillo (2009) determinaron que las ninfas de la mosca pinta tienen una distribución binomial negativa; es decir, los insectos tienen una disposición espacial en agregados. Esta disposición espacial se debe principalmente a factores bióticos y abióticos, como la precipitación y la temperatura (Castro et al., 2005). También se ha estudiado la disposición espacial en relación a la dinámica poblacional (Pires et al., 2000; Castro et al., 2005). Se ha propuesto el control de maleza como los zacates Pangola Digitaria decumbens Steud (1930), Johnson Sorghum halepense (L.) Pers. 1805, y Privilegio Panicum maximum Jacq. 1781, para eliminar otras hospederas naturales de la mosca pinta y así reducir el nivel de infestación (Enríquez et al.,
1999). Otros trabajos se han orientado a evaluar la rastra fitosanitaria, el aporque y la requema (Badilla, 2002), así como el control químico de las malezas con la aplicación de ingredientes activos como ametrina, atrazina, glifosato, paraquat, y 2,4-D (Perdomo, 2004). Además, se han realizado estudios relacionados con el muestreo de los huevos, ya que su control se considera como una práctica preventiva para reducir la incidencia de adultos en años posteriores, además de obtener información de las poblaciones de la plaga para mejorar la toma de decisiones en los métodos de control a utilizar (Canela-Cantellano, 2015).

En el caso del control químico, se han evaluado diferentes dosis y métodos de aplicación con insecticidas organosintéticos de varios grupos toxicológicos e ingredientes activos, tales como organoclorados, organofosforados, piretroides y carbamatos (Flores, 1994). En cuanto al control biológico, se han realizado estudios con virus, bacterias, enemigos naturales, nematodos y hongos contra la plaga (Van Driesche \& Bellows, 1996). Flores et al. (1965) indicaron que la cría artificial y liberación de los hemípteros Zelus luridus Stål 1862 y Castolus plagiaticollis Stål 1858, no tuvieron el control esperado. Aunque Ferrer et al. (2004) han reportado que la aplicación de entomopatógenos como Metarhizium anisopliae (Metschnikoff) Sorokin, 1883 y Beauveria bassiana (Bals. Criv.) Vuill. 1912 controlan las poblaciones de esta plaga, lo que actualmente se considera una medida de control de uso común.

Existen diferentes alternativas para evaluar el efecto simultáneo de varios factores en la dinámica poblacional de los insectos; se pueden emplear modelos de simulación (Acuña \& Barnichi, 1996) o modelos de regresión (Valdez-Torres et al., 2012), entre otros. García-García et al. (2006) estudiaron algunos factores bióticos y abióticos relacionados con la mosca pinta en un modelo predictivo de riesgo, y evaluaron de manera probabilística su contribución a la presencia de poblaciones dañinas de esta plaga. También se han empleado modelos lineales mixtos, que consideran efectos fijos como aleatorios en plagas como Neochetina eichhorniae Warner 1970, Liometopum apiculatum Mayr 1870 (Velasco-Corona et al., 2007; Solange-Martínez et al., 2013). En el caso de las poblaciones de adultos de mosca pinta, es de interés evaluar el efecto de los factores bióticos, abióticos y agronómicos de manera conjunta. Por lo que el objetivo de este trabajo fue cuantificar la influencia de algunos factores que inciden en la ocurrencia de adultos de la mosca pinta. 


\section{MATERIALES Y MÉTODOS}

Área de estudio. Se seleccionaron quince parcelas que proveen de materia prima a los ingenios azucareros "El Potrero" y "La Gloria" en el estado de Veracruz. Estas parcelas se georreferenciaron y se les asignó un código para su identificación con el apoyo del personal técnico de los ingenios, se calificaron sus niveles de infestación del año previo (Cuadro 1): mayores (A) o menores (B) al umbral económico; el umbral económico considerado fue de 0.2 ninfas tallo-1 y 0.2 adultos tallo $^{-1}$ (Gómez, 2007; INIFAP, 2014).

Muestreo e identificación de especies. Se realizaron conteos y mediciones de algunos factores que se supusieron afectan las poblaciones de adultos de mosca pinta. Los factores bióticos fueron: poblaciones de ninfas, cobertura de maleza y nivel de infestación de adultos del año previo. Los factores abióticos fueron temperatura y precipitación; también se evaluaron factores de manejo agronómico como la aplicación de insecticidas y de entomopatógenos, y el tiempo de muestreo. El registro de las variables anteriores se llevó a cabo durante muestreos semanales de adultos y ninfas de mosca pinta del 2 de julio al 15 de octubre de 2014, que corresponde a los meses con mayores poblaciones (Flores, 1994; García-García et al., 2006). Para realizar el muestreo de adultos se colocaron 40 trampas

Cuadro 1. Geolocalización de las parcelas inspeccionadas por ingenio azucarero. El nivel de infestación se refiere a la densidad de mosca pinta por arriba (A) o abajo (B) del umbral económico en el año previo.

\begin{tabular}{ccccc}
\hline Ingenio & Clave & Latitud & Longitud & Nivel de infestación \\
\hline E1 Potrero & P01 & 18.9052 & -96.8530 & A \\
& P02 & 18.8555 & -96.8266 & A \\
& P03 & 18.8210 & -96.8374 & A \\
& P04 & 18.8968 & -96.7807 & A \\
& P05 & 18.8197 & -96.7012 & B \\
& P06 & 18.8898 & -96.7788 & A \\
& P07 & 18.9040 & -96.7883 & B \\
& P08 & 18.8731 & -96.8708 & B \\
& P09 & 18.8227 & -96.7347 & B \\
& P10 & 18.8831 & -96.7881 & B \\
La Gloria & G01 & 19.3847 & -96.4296 & A \\
& G02 & 19.3904 & -96.4329 & A \\
& G03 & 19.3076 & -96.3886 & A \\
& G04 & 19.2314 & -96.369 & B \\
& G05 & 19.2115 & -96.3807 & B \\
\hline
\end{tabular}

amarillas de cartulina de $14 \times 21 \mathrm{~cm}$ con pegamento Stickbug ${ }^{\circledR}$; se distribuyeron 20 en el interior y 20 en los bordes de la parcela a una altura aproximada de $1.50 \mathrm{~m}$ a nivel del suelo. Las trampas se retiraron a las $24 \mathrm{~h}$ y se llevaron al laboratorio donde se realizó la identificación de las especies con las claves taxonómicas de Thompson \& León (2005). Las ninfas fueron contabilizadas mediante inspección visual en un metro cuadrado, considerando $0.5 \mathrm{~m}$ de cada lado del surco. Se utilizaron los mismos sitios donde se colocaron las trampas amarillas para la captura de los adultos. Para calcular la cobertura de maleza tanto en el interior como en los bordes de las parcelas, se utilizó una escala categórica con valores que se agrupan de 0 a $25 \%$ (A), 26 a $50 \%$ (B) y 51 a $75 \%$ (C), respectivamente. La inspección visual de la cobertura de maleza se realizó en un metro cuadrado y en ambos casos se utilizaron los mismos sitios donde se colocaron las trampas amarillas. Las parcelas se clasificaron en aquellas que recibieron control químico o control biológico para combatir la mosca pinta. El control químico consistió en la aplicación de la mezcla insecticida de Tiametoxam + Lambdacihalotrina $(10.6 \%$ $+14.1 \%$ ) a una dosis de $300 \mathrm{ml} /$ ha en las parcelas G01 y G05 del ingenio la Gloria y de Bifentrina + Imidacloprid $(4.5 \%+22.4 \%)$ a una dosis de $500 \mathrm{ml} /$ ha en las parcelas P01, P05, P06, P08 y P09 del ingenio El Potrero (Cuadro 1). El control biológico consistió en la aplicación de $M$. anisopliae a una dosis de $200 \mathrm{~g} / \mathrm{ha}$ aplicado en la parcela $\mathrm{P} 03$. En los tres casos fueron aplicaciones únicas durante el mes de agosto. Además, para incluir temperatura y precipitación en el modelo, se compiló la información climática de las estaciones meteorológicas de ambos ingenios azucareros. Se utilizó la precipitación acumulada semanal previa a cada fecha de muestreo, así como la temperatura promedio en ese intervalo.

Análisis estadístico. Para cuantificar la contribución de los factores que influyen en la presencia de adultos de la mosca pinta, se estimaron los parámetros de un modelo lineal mixto. El modelo empleado para analizar los factores estudiados fue, en la notación del lenguaje R:

ADULTO INFEST + NINFA + CBI + CBE + DJUL + APINS + APENT + PPT + TEMP (1|PARCELA/TRAMPA)

Este modelo representa el efecto aditivo lineal en los conteos de adultos de mosca pinta ADULTO de los factores a la derecha de la tilde $(\sim)$, es decir, en función del nivel de infestación del año previo INFEST, las poblaciones de ninfas NINFA, la cobertura interna de maleza $\mathrm{CBI}$, la cobertura de maleza en los bordes CBE, el tiempo de muestreo DJUL, la aplicación de insecticidas APINS 
y entomopatógenos APENT, la precipitación acumulada de la semana previa PPT y la temperatura promedio de la semana previa TEMP. Este modelo es mixto, pues considera tanto efectos fijos como aleatorios (Faraway, 2006). Los efectos fijos son los del lado derecho de la tilde, excepto los componentes en paréntesis. Se consideró que las parcelas PARCELA son una muestra aleatoria de las posibles parcelas en la zona, lo mismo que las trampas TRAMPA, que se encuentran anidadas en las parcelas. Las variables y sus unidades de medida se enlistan en el Cuadro 2. Para construir intervalos de confianza de los parámetros del modelo y probar su significancia, se hicieron 1000 corridas adicionales con muestreos bootstrap paramétrico con la función confint(). Adicionalmente, se hicieron 100 corridas con bootstrap no paramétrico para visualizar la variabilidad en las estimaciones. Los intervalos de confianza (IC95) se calcularon con los cuartiles $(0.025,0.975)$ para cada parámetro del modelo (Bolker et al., 2008). Mientras más alejado del cero estén los valores de los estimadores, se considera mayor su efecto sobre las poblaciones de adultos de mosca pinta; si los valores cruzan el cero, se considera que no hay efecto significativo. Los parámetros del modelo lineal mixto se estimaron con la función $\operatorname{lmer();~los~análisis~se~hicieron~con~el~programa~}$ R v. 3.2.1. (R Core Team, 2015).

\section{RESULTADOS}

Especies de mosca pinta. Durante el periodo del 2 de julio al 15 de octubre, se capturaron 14623 adultos de mosca pinta y se contabilizaron 6826 ninfas. Del total de los adultos capturados, la especie en mayor número fue Aeneolamia contigua (Walker, 1851) con 14341 (98\%), seguida de Aeneolamia albofasciata (Lallemand, 1939) con 269 (1.8\%) y Prosapia simulans (Walker, 1858) con 13 ejemplares $(<1 \%)$.

Importancia relativa de los componentes del modelo que afectan las poblaciones de mosca pinta. En la Figura 1 se observa la distribución del muestreo de los parámetros del modelo, que toman valores aproximados entre -0.5 y 3.5. El nivel de infestación en el año previo es relevante para definir las poblaciones actuales de adultos IC95 (-0.393, -0.108); los valores negativos se deben a que se compara el nivel bajo de infestación contra el nivel alto de infestación que es el nivel base (B-A), con un coeficiente de variación de $22.2 \%$. La variable NINFA corresponde al efecto de las poblaciones de ninfas y se observa que afectan positivamente las poblaciones de adultos IC95 $(1.90,1.94)$ con $\mathrm{CV}=1.8 \%$; en este caso su variabilidad es reducida, lo que indica una estrecha relación con las poblaciones de adultos. En cuanto a la cobertura de maleza en el interior, los parámetros indican la diferencia entre el nivel correspondiente y el nivel base, ya que IB-IA mide la diferencia entre el nivel IB de cobertura de maleza contra el nivel IA y aparece como significativo: IC95 IB- IA $(0.255,0.525)$ con $\mathrm{CV}=$ $33.6 \%$, e IC-IA $(0.96,1.83)$ con $\mathrm{CV}=41.3 \%$. La misma interpretación se aplica a la cobertura en los bordes, la cual presenta valores negativos en relación la diferencia EB- EA, lo que indica menos capturas, mientras que la diferencia EC- EA presenta valores positivos, señalando

Cuadro 2. Componentes del modelo lineal mixto para evaluar el efecto de diferentes factores sobre las poblaciones de adultos (ADULTO) de mosca pinta.

\begin{tabular}{lll}
\hline Componente & Descripción & Unidad (niveles) \\
\hline ADULTO & Población de adultos de mosca pinta & Adultos trampa ${ }^{-1}$ \\
INFEST & Densidad previa: baja (B) o alta (A) de la parcela & Binaria (B, A) \\
NINFA & Población de ninfas de mosca pinta & Ninfas m $^{-2}$ \\
CBI & Cobertura de maleza en el interior de la parcela & Categórica (IA, IB, IC) \\
CBE & Cobertura de maleza en el borde de la parcela & Categórica (EA, EB, EC) \\
DJUL & Tiempo de muestreo & Día juliano \\
APINS & Aplicación de insecticidas en la parcela & Binaria (IS, IN) \\
APENT & Aplicación de entomopatógenos en la parcela & Binaria (ES, EN) \\
PPT & Precipitación acumulada de una semana previa & mm \\
TEMP & Temperatura media (promedio de la semana previa) & ${ }^{\circ} \mathrm{C}$ \\
PARCELA & Parcelas de la zona & Categórica \\
TRAMPA & Código de la trampa & Categórica \\
\hline
\end{tabular}




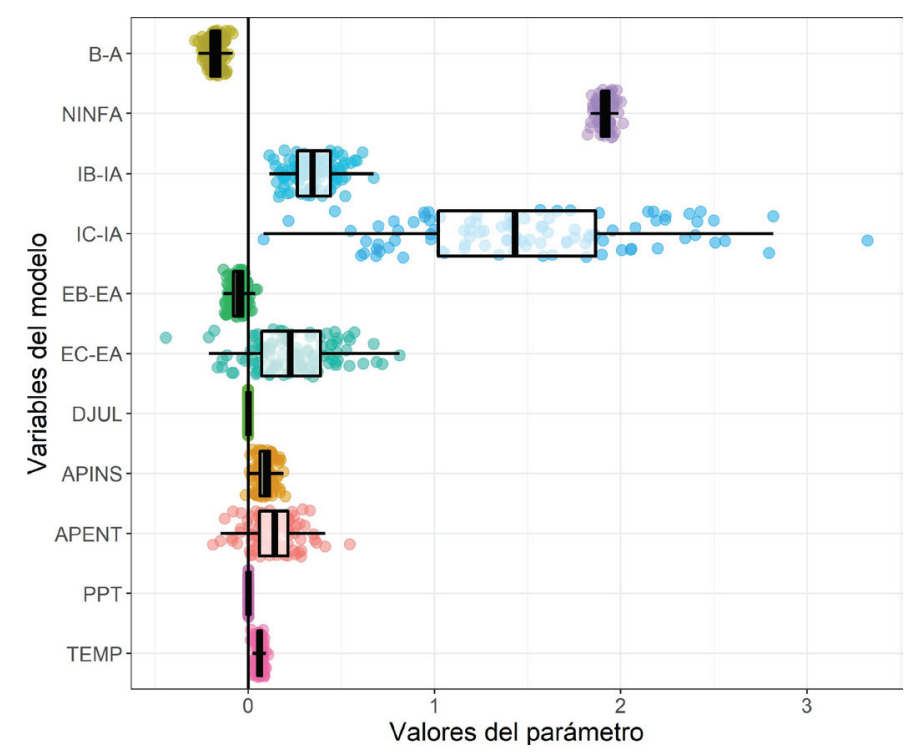

Figura 1. Distribución de muestreo bootstrap (puntos) y gráficas de caja de los parámetros del modelo lineal mixto que representa las poblaciones de adultos de la mosca pinta en caña de azúcar de Veracruz, México. Las líneas de las gráficas de caja corresponden a los valores extremos y el rectángulo delimita el 25, 50 y $75 \%$ percentil de la distribución del parámetro respectivo.

más capturas de mosca pinta que el nivel base: IC95 EBEA $(-0.222,0.066)$ con $C V=69 \%$, EC-EA $(-0.358,0.678)$ con $\mathrm{CV}=95 \%$ respectivamente. Comparativamente, es más pronunciado y consistente el efecto de la cobertura de maleza en el interior de las parcelas que en los bordes. Por otra parte, la aplicación de insecticidas y entomopatógenos no tienen un efecto significativo en la captura de mosca pinta, y su efecto es menor a los factores previos. Se observa mayor variabilidad en la respuesta a los entomopatógenos que a los insecticidas: IC95 APINS (-0.133, $0.329)$ con $\mathrm{CV}=46.3 \%$ e IC95 APENT $(-0.328,0.623)$ con $\mathrm{CV}=92.7 \%$. Los estimadores para el tiempo IC95 ($0.0022,0.0004)$ con $\mathrm{CV}=41.4 \%$ y la precipitación IC95 $(-0.001,0.003)$ con $\mathrm{CV}=60.3 \%$, no indican un efecto en las poblaciones de adultos, mientras que la temperatura tiene un efecto relativamente menor pero significativo: IC95 TEMP $(0.009,0.106)$ con un $\mathrm{CV}=30.7 \%$. Los valores del intercepto se omiten para visualizar mejor el efecto de los parámetros asociados a los factores estudiados y fueron en su mayoría negativos, con IC95 (-2.676, 0.216). En resumen, se observó un efecto significativo positivo de las ninfas, la cobertura de malezas interna y la temperatura en las capturas de adultos de la mosca pinta, siendo el mayor efecto el de las ninfas y el de la presencia de malezas en la zona interna de la parcela.

\section{Efecto de los factores involucrados en las poblaciones de adultos de mosca pinta}

Factores bióticos. En el caso de las ninfas, en la Figura 2 se observa la relación entre los adultos capturados y las ninfas observadas en cada una de las parcelas. Es claro que las poblaciones de adultos dependen significativamente de las poblaciones de las ninfas, lo que se refleja en los altos valores del parámetro asociado a este factor en el modelo mixto, ya que, en promedio, hay un incremento de capturas de 1.92 adultos por trampa por ninfa (Fig. 1). Solamente en las parcelas P08 y P09 se observó una relación menos clara entre las ninfas y los adultos, debido a las bajas poblaciones presentes.

Por otra parte, en la Figura 3 se observa el efecto del nivel de infestación del año previo en las poblaciones de adultos en el período de estudio. El promedio de adultos capturados aumenta en relación con la alta densidad poblacional del año previo en 1.48 adultos por trampa. Esto señala que la cantidad de huevos depositados por las poblaciones del año previo sí influyen significativamente en las poblaciones del año siguiente, de acuerdo con el análisis del modelo.

En cuanto a la cobertura de malezas en el interior de las parcelas en la Figura 4 se muestra la relación con los promedios de adultos capturados. Se observa que a medida que existe mayor cobertura de maleza, la población de adultos de mosca pinta aumenta. De acuerdo con esta figura y los resultados del modelo se observa que las ca-

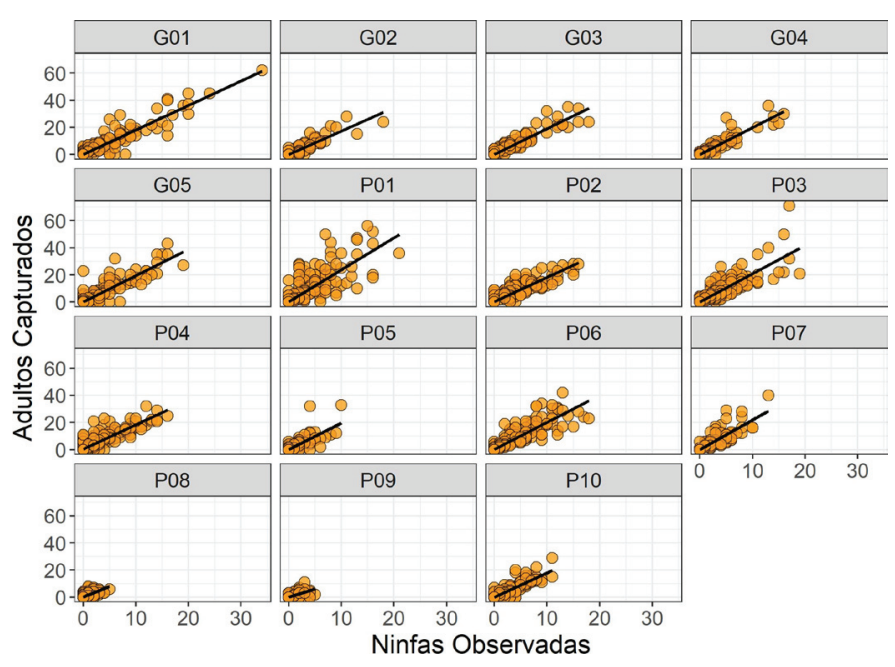

Figura 2. Relación entre los conteos de ninfas y los adultos de mosca pinta capturados en 15 parcelas de caña de azúcar de Veracruz, México. 


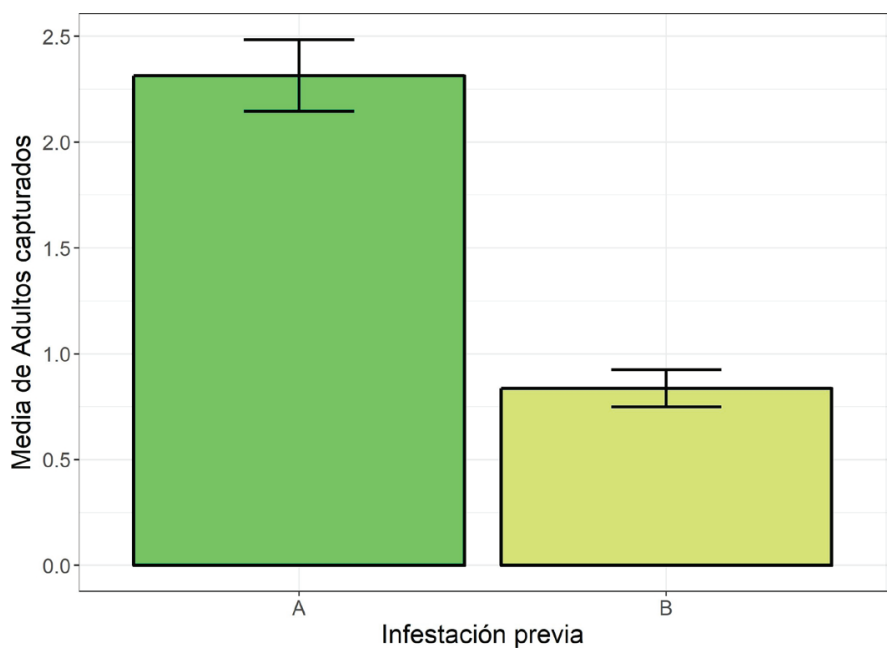

Figura 3. Densidad poblacional de mosca pinta en parcelas de caña de azúcar con alta (A) y baja densidad (B) del año previo, la línea vertical indica el intervalo de confianza del $95 \%$.

tegorías IB e IC difieren significativamente del nivel más bajo de cobertura (IA), pero son similares entre sí (Fig. 1), el incremento en el número de adultos capturados es de $3 \mathrm{y}$ 4.5 por trampa para los niveles B y C respectivamente. En la Figura 5 se ilustra la cobertura de la maleza en el exterior de la parcela y el promedio de adultos capturados. Las capturas de adultos cuando hubo mayor cobertura de maleza (EC) no difieren de los valores cuando la cobertura es

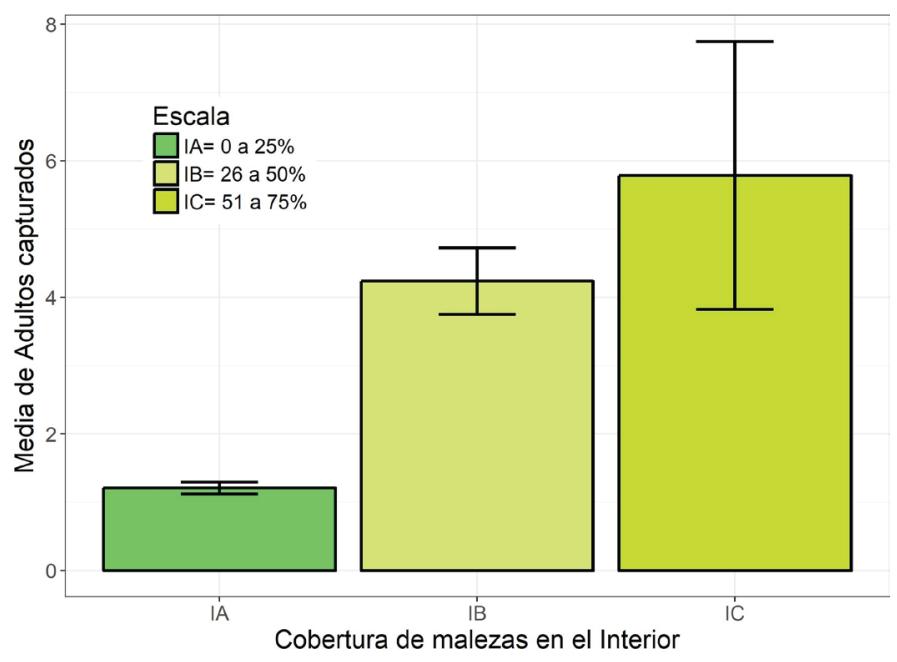

Figura 4. Nivel de cobertura de maleza en el interior de las parcelas de caña de azúcar en relación con el promedio de adultos de mosca pinta capturados, utilizando una escala categórica donde los valores son IA 0 a $25 \%$, IB 26 a $50 \%$ e IC 51 a $75 \%$ de cobertura, la línea vertical indica el intervalo de confianza de $95 \%$. mínima (EA). La categoría EB sí disminuye ya que tiene poblaciones menores de adultos en relación a la categoría EA. Esta fluctuación podría deberse a que las parcelas en estudio tuvieran un intercambio de adultos con parcelas adyacentes. Sin embargo, de acuerdo con el análisis del modelo mixto, estas diferencias no son significativas. Factores abióticos. La Figura 6 ilustra el promedio acumulado de la precipitación de la semana previa al mues-

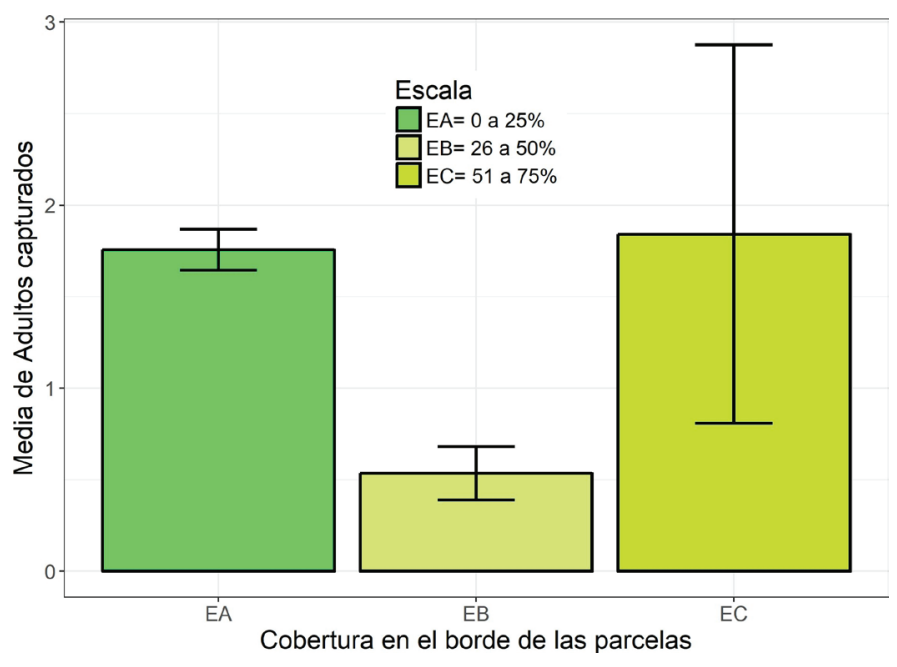

Figura 5. Nivel de cobertura de maleza en el exterior de las parcelas de caña de azúcar en relación con los promedios de adultos de mosca pinta capturados, utilizando una escala categórica donde los valores son EA 0 a 25\%, EB 26 a 50\% y EC 51 a $75 \%$ de cobertura, la línea vertical indica el intervalo de confianza del 95\%.

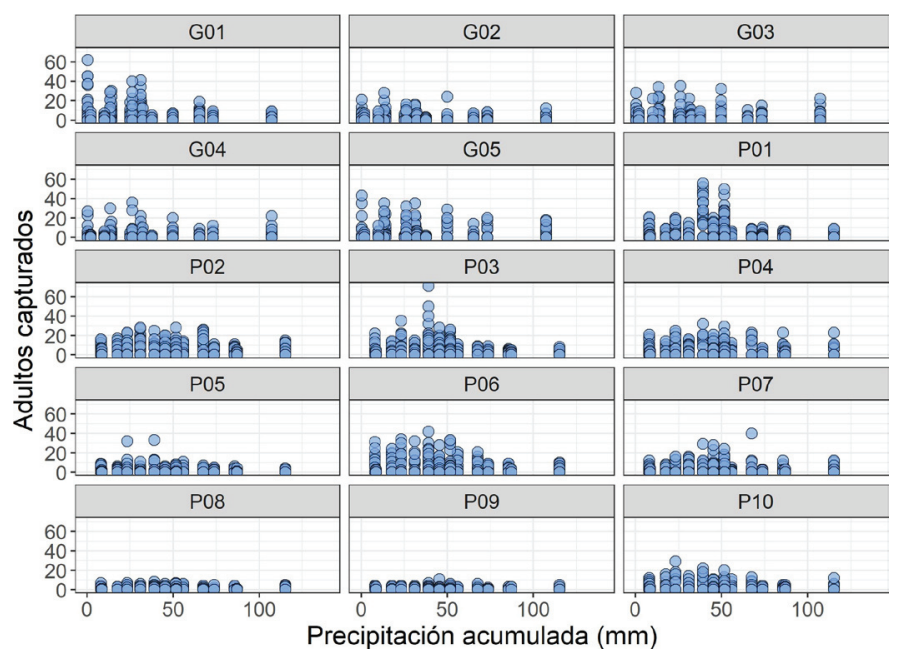

Figura 6. Relación de la precipitación acumulada con los adultos de mosca pinta capturados en parcelas de caña de azúcar en Veracruz, México. 
treo y la relación con los adultos capturados en las trampas amarillas. En este caso, las poblaciones de adultos no se ven afectadas significativamente, como se observa en el análisis del modelo mixto (Fig.1). En el caso de la temperatura, la Figura 7 muestra la media acumulada en la semana previa al muestreo y su relación con los adultos capturados, con un efecto significativo pero muy reducido (Fig. 1).

En el caso del tiempo de muestreo, la Figura 8 muestra las fechas y su relación con los adultos capturados, allí se observa una fluctuación esencialmente unimodal, siendo el pico de mayor captura el día juliano 218 (6 de agosto) con más de 15 adultos promedio por captura por trampa. En general, las densidades más altas ocurrieron entre los días julianos 204 (23 de junio) al 239 (27 de agosto); no obstante, el análisis estadístico no indicó un efecto significativo.

\section{DISCUSIÓN}

Los resultados del modelo lineal mixto cuantifican el grado de influencia de cada una de las variables sobre las capturas de adultos de la mosca pinta en la caña de azúcar. La infestación registrada en el año previo es un factor que influye en las altas densidades poblacionales de adultos presentes, lo cual también fue reportado por García-García et al. (2006), quienes mencionan que este factor puede estar relacionado con el umbral económico, tal y como lo indican Gómez (2007) e INIFAP (2014). Otro factor

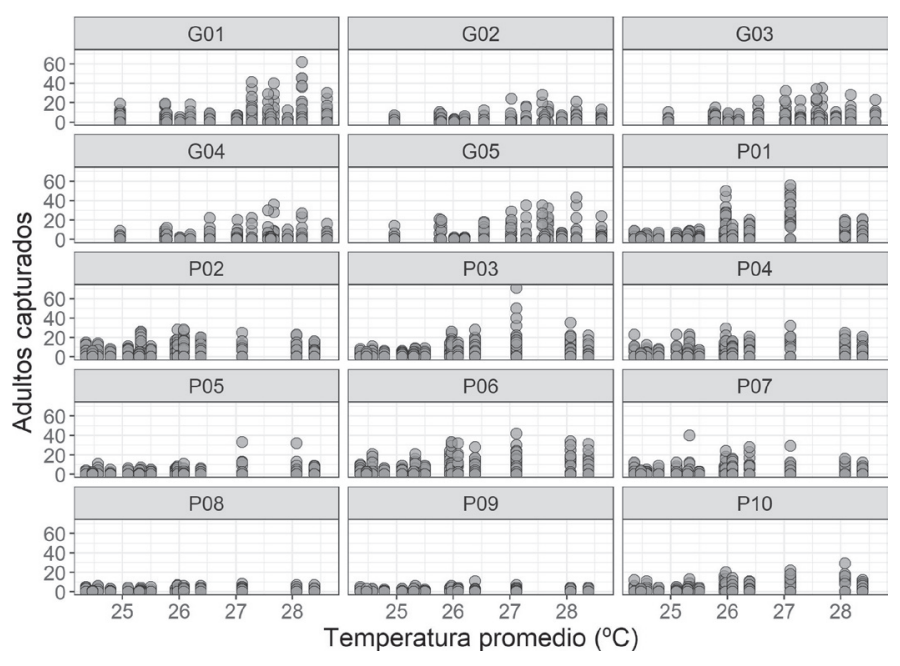

Figura 7. Relación de la temperatura promedio $\left({ }^{\circ} \mathrm{C}\right)$, con los adultos de mosca pinta capturados en caña de azúcar en Veracruz, México. relacionado con las poblaciones presentes de adultos es la densidad poblacional de los huevos de los años previos (Auad et al., 2011). Aunque no hacen referencia directa a los huevos, García-García et al. (2006) mencionan que uno de los factores más importantes para la incidencia de la mosca pinta son las ninfas, que pueden provenir de huevos invernantes del año previo, ya que como se observa en el modelo, existe una estrecha relación con las poblaciones de adultos. Castro et al., (2005) mencionan que un indicador de la presencia de adultos presentes en la parcela son las ninfas. Además, se ha encontrado que las ninfas de Aeneolamia spp., también causan daños en la caña de azúcar (Badilla, 2002).

En el presente estudio no se encontró una relación clara entre la precipitación y el muestreo de adultos, posiblemente debido a que se tuvieron rangos de precipitación reducidos, relativos al corto periodo en que se realizó el muestreo. Lo anterior a pesar de que se sabe que la precipitación afecta a las poblaciones de adultos, pues en la época de sequía las poblaciones se reducen (Flores et al., 1965); sin embargo, en el período de muestreo se presentaron lluvias, por lo que este escenario no ocurrió. Por otra parte, varios autores han registrado un efecto positivo de la precipitación en la incidencia de la mosca pinta (García-García et al., 2006; Figueredo et al., 2012). El CIAT (1982) relaciona la incidencia de las ninfas con la humedad relativa y la eclosión de los huevecillos diapáusicos. Otros autores mencionan que, de acuerdo con la especie y las condiciones ambientales de la región, la mosca pinta puede presentar cinco o más generaciones por año (Badilla, 2002). Varios estudios reportan que las temperaturas inciden en la emergencia de las ninfas de mosca pinta en primavera (Valerio \& Koller, 1993; López-Collado et al., 2003). Aunque en este estudio el efecto de la temperatura fue relativamente menor, esto pudo deberse al rango reducido en los valores de la temperatura y al tiempo de muestreo.

En el caso del efecto de la maleza en el interior como en los bordes de las parcelas, en general éstas fomentaron la ocurrencia de adultos de mosca pinta. García-García et al. (2006) mencionan que la presencia de maleza es uno de los factores más importantes para la incidencia de la mosca pinta. Otros autores indican que algunas las malezas son plantas hospederas naturales de la mosca pinta (Enríquez et al., 1999). Sáenz et al. (1999) comentan que se debe realizar un adecuado control de maleza, incluyendo caminos interiores y el contorno de las parcelas, para impedir la migración de los insectos a otras parcelas.

Las aplicaciones de insecticidas y entomopatógenos fueron variables que no presentaron un efecto significati- 


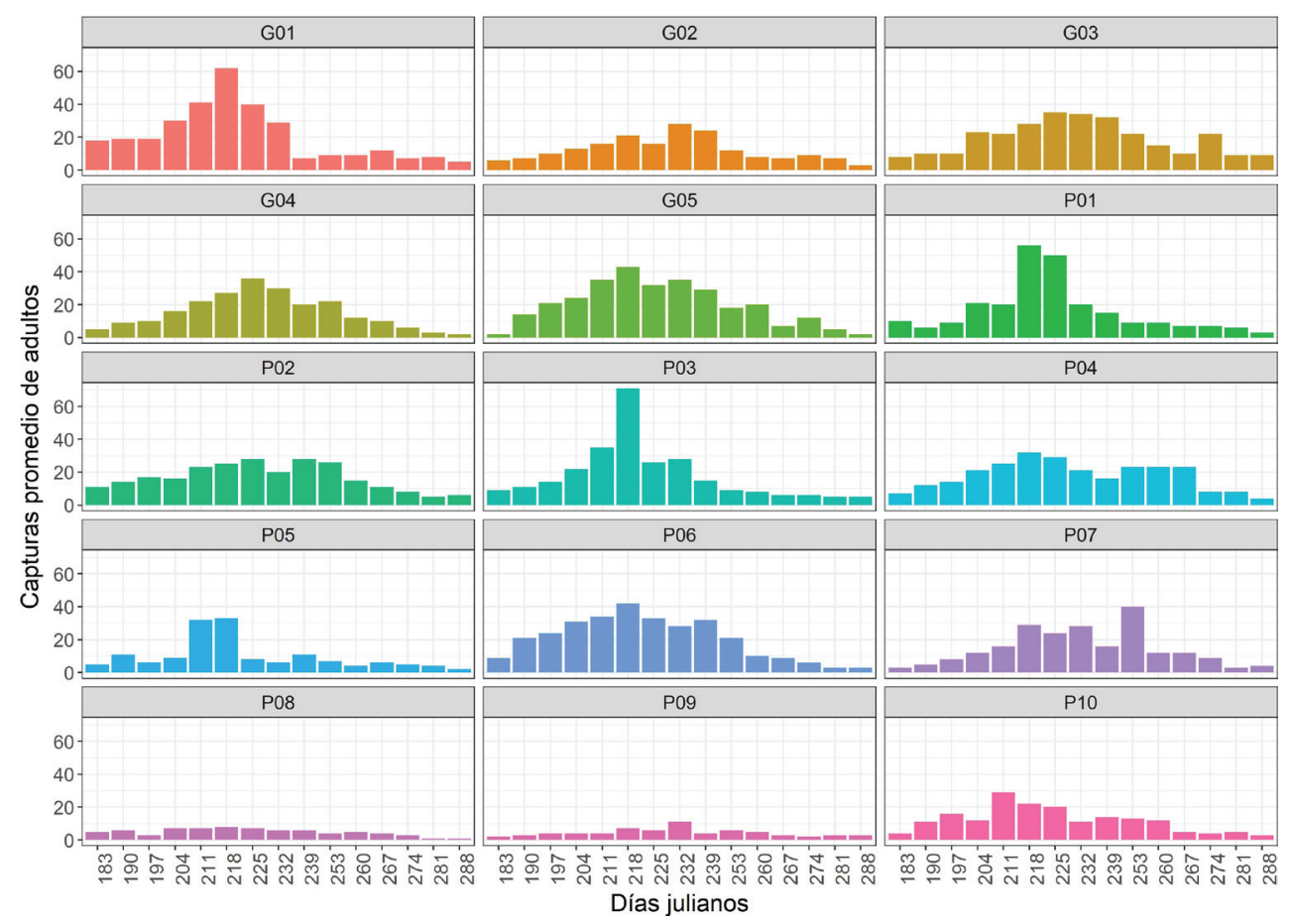

Figura 8. Relación de las fechas de muestreo con la distribución temporal de los adultos capturados en 15 parcelas de caña de azúcar en Veracruz, México.

vo en las capturas de adultos. Esto pudo deberse al corto tiempo que dura el efecto de los productos, ya que cuando éste termina, emergen más individuos. El control de ninfas y adultos puede realizarse con métodos biológicos, donde M. anisopliae y B. bassiana (Bautista-Gálvez \& González-Cortes, 2005) son opciones relevantes. Sin embargo, los ingenios aun consideran a los productos químicos como un método de control importante para disminuir las densidades poblacionales de la mosca pinta (DinardoMiranda et al., 2004), aunque sus efectos sean cortos.

\section{CONCLUSIONES}

Las variables que se relacionaron positivamente con las poblaciones de adultos de mosca pinta fueron la presencia de ninfas, el nivel de infestación de adultos en la temporada previa, la cobertura de maleza en el interior de la parcela y la temperatura. Las ninfas contribuyen en un incremento de 1.92 adultos por trampa, infestaciones altas en el año previo contribuyen con 1.48 adultos por trampa, la cobertura de malezas contribuye entre 3 y 4.5 adultos por trampa y la temperatura en 0.051 adultos por trampa. La presencia de maleza en los bordes afectó irre- gularmente las poblaciones de adultos, posiblemente por el intercambio de ellos con las parcelas adyacentes y su efecto no fue significativo. Finalmente, la precipitación y el tiempo de muestreo tampoco tuvieron efecto en las poblaciones de adultos durante el período de estudio.

AGRADECIMIENTOS. Al Consejo Nacional de Ciencia y Tecnología, por la beca de maestría otorgada a JCGG. Al ingeniero Luis Arturo Martínez Rivera, Superintendente Técnico de Campo del ingenio El Potrero, y al Ingeniero Leopoldo Montero Ramírez jefe del departamento de plagas del ingenio la Gloria por su apoyo en la logística del proyecto. A dos árbitros anónimos por sus valiosas sugerencias para mejorar el artículo.

\section{LITERATURA CITADA}

Acuña, S. T. \& Barnichi, de G. G. (1996). Modelo de simulación para el manejo integrado de las plagas de ecosistemas agrícolas. Boletín de Sanidad Vegetal Plagas, 22, 761-773.

Auad, A. M., Simoes, A. D., Leite, M. V., da Silva, S. E. B., dos Santos, D. R. \& Monteiro, P. H. (2011). Seasonal dynamics of egg diapause in Mahanarva spectabilis (Distant, 1909) (Hemiptera: Cercopidae) on elephant grass. Arquivos do Instituto Biológico, 78, 325-330.

Badii, M. H. \& Castillo, J. V. (2009). Distribuciones probabilísticas 
de uso común. Daena: International Journal of Good Conscience, 4, 149-178.

Badilla, F. F. (2002). Un programa exitoso de control biológico de insectos plaga de la caña de azúcar en Costa Rica. Manejo Integrado de Plagas y Agroecología, 64, 77-87.

Bautista-Gálvez, A. \& González-Cortes, N. (2005). Tres dosis de Metarhizium anisopliae sobre la mosca pinta (Aeneolamia spp.) en caña de azúcar en la región de los ríos, estado de Tabasco Universidad y Ciencia, 21, 37-40.

Bolker, B. M., Brooks, M. E., Clark, C. J., Geange, S. W., Poulsen, J. R., Stevens, M. H. H. \& White, J. S. S. (2008). Generalized linear mixed models: a practical guide for ecology and evolution. Trends in Ecology and Evolution, 24, 127-135.

Canela-Cantellano, J. (2015). Métodos de muestreo para la detección de huevos de mosca pinta Aeneolamia albofasciata (Lallemand) en caña de azúcar. Tesis de Maestría en Ciencias. Colegio de Postgraduados. Campus Veracruz. $70 \mathrm{p}$.

Castro, U., Morales, A. \& Peck, D. C. (2005). Dinámica poblacional y fenología del salivazo de los pastos Zulia carbonaria (Lallemand) (Homoptera: Cercopidae) en el Valle Geográfico del Río Cauca, Colombia. Neotropical Entomology, 34, 459-469.

CIAT, (1982). Programa de pastos tropicales; Informe anual. Centro Internacional de Agricultura Tropical, Cali. 28 p.

De la Cruz, L. J. J., Vera-Graziano, J., López-Collado, J., Pinto, V. M. \& Garza-García, R. (2005). Una técnica simple para el desarrollo de ninfas de Aeneolamia postica (Homoptera: Cercopidae). Folia Entomológica Mexicana, 44, 91-93.

Dinardo-Miranda, L. L., Coelho, A. L., \& Ferreira, J. M. G. (2004). Influência da época de aplicação de inseticidas no controle de $\mathrm{Ma}$ hanarva fimbriolata (Stål) (Hemiptera: Cercopidae), na qualidade e na produtividade da cana-de-açúcar. Neotropical Entomology, 33, 91-98.

Enríquez, Q. J. F., Meléndez, N. F. \& Bolaños, A. E. D. (1999). Tecnología para la producción y manejo de forrajes tropicales en México. INIFAP. CIRGOC. Campo Experimental Papaloapan. Libro Técnico No. 7. Veracruz, México. 262 p.

Evans, D. E. (1972). The spatial distribution and sampling of Aeneolamia varia saccharina and $A$. postica jugata (Homoptera: Cercopidae). Entomología Experimentalis \& Applicata, 15, 305-318.

Faraway, J. (2006). Extending the linear model with R. Generalized lineal, mixed effects and nonparametric regression models. Chapman \& Hall/CRC, Boca Raton. 322 p.

Ferrer, F., Arias, M., Trelles, A., Palencia, G., Navarro, J. M. \& Colmenares, R. (2004). Posibilidades del uso de nematodos entomopatógenos para el control de Aeneolamia varia en caña de azúcar. Manejo Integrado de Plagas y Agroecología, 72, 39-43.

Figueredo, L., Andrade, O., Cova, J., Mora, O. \& Aza, G. (2012). Distribución espacio temporal de ninfas de Aeneolamia varia Fabricius (1787) (Hemiptera: Cercopidae) en caña de azúcar a través de un sistema de información geográfica. Entomotropica, 21, 718 .

Flores, C. S. (1994). Las plagas de la caña de azúcar en México. IMPA. México D.F. 350 p.

Flores, C. S. (1996). Mosca Pinta o Salivazo en Caña de Azúcar y Pastos. In: Memorias del primer Encuentro Regional Fitosanitario. Colegio de Postgraduados - GEPLACEA - CyTCAÑA, Córdoba, Ver. México. 4 p.
Flores, C. S., Ramírez, M. A. \& Cortés, I. A. (1965). El salivazo de la caña de azúcar. Instituto para el Mejoramiento de la Producción de Azúcar. Boletín de Divulgación No. 5. México. 70 p.

García-García, C. G., López-Collado, J., Nava-Tablada M. E., Villanueva-Jiménez, J. A. \& Vera-Graziano, J. (2006). Modelo de predicción de riesgo de daño de la mosca pinta Aeneolamia postica (Walker) Fennah (Hemiptera: Cercopidae). Neotropical Entomology, 35: 677-688.

Gómez, L. A. (2007). Manejo del salivazo Aeneolamia varia en cultivos de caña de azúcar en el valle del río Cauca. Cenicaña (Colombia), Carta Trimestral, 29, 10-17.

INEGI, (2014). Instituto Nacional de estadística y Geografía. Available at: http://www.inegi.org.mx/est/contenidos/Proyectos/encuestas/agropecuarias/ena/Ena2014/default_t.aspx (accessed on September 2015).

INIFAP, (2014). Trasferencia de tecnología de la dinámica poblacional y control integrado del barrenador y mosca pinta de la caña de azúcar. Available at: http://biblioteca.inifap.gob. mx:8080/xmlui/bitstream/handle/123456789/3821/MOSCAPINTA_CA\%C3\%91A_REYESA.pdf?sequence $=1$ (accessed on May 2016).

López-Collado, J., Bravo, M. H., Vera, G. J. \& Villanueva, J. J. A. (2003). APROBAR: Programa de cómputo para el análisis probabilístico de riesgos de plagas agrícolas. Entomología Mexicana, 2, 448-449.

Peck, D. C. (2001). Diversidad y distribución geográfica del salivazo (Homoptera: Cercopidae) asociado con gramíneas en Colombia y Ecuador. Revista Colombiana de Entomología, 27, 129-136.

Perdomo, R. F. (2004). Dinámica de la flora arvense de caña de azúcar en Tlaquiltenango, Morelos, México. Tesis de Doctorado. Colegio de Postgraduados, Campus Montecillo. Texcoco, México. 102 p.

Pires, C. S. S., Price, P. W. \& Fontes, E. G. (2000). Preference performance linkage in the neotropical spittlebug Deois flavopicta, and its relation to the phylogenetic constraints hypothesis. Ecological Entomology, 25, 71-80.

R Core Team. (2015). R: A language and environment for statistical computing. R Foundation for Statistical Computing, Vienna, Austria. http://www.R-project.org/.

Sáenz, C., Alfaro, D., Salazar, J. D., Rodríguez, A., \& Oviedo, R. (1999). Evolución histórica del manejo de plagas en el cultivo de la caña de azúcar en Costa Rica. In: XI Congreso Nacional Agronómico / V Congreso Nacional de Entomología. San José, Costa Rica. 147-153 p.

Salazar, B., J. D. \& Badilla, F. F. (1997). Evaluación de dos cepas del hongo entomopatógeno Metarhizium anisopliae y seis insecticidas granulados en el control de salivazo (Aeneolamia postica) (Homoptera: Cercopidae) en caña de azúcar en la región de San Carlos, Costa Rica. Manejo Integrado de Plagas y Agroecología, 43, 9-18.

Salgado, G. S., Bucio, A. L., Riestra, D. \& Lagunes-Espinoza, L. C. (2003). Caña de Azúcar: Hacia un manejo sustentable. Colegio de Postgraduados, Campus Tabasco. México. 384 p.

SIAP. (2017). Anuario estadístico de la producción agrícola. Available at: http://infosiap.siap.gob.mx/aagricola_siap_gb/icultivo/index.jsp/ (accessed on August 2017).

Solange-Martínez, F., Franceschini, M. C., \& Poi, A. (2013). Preferencia alimentaria de Neochetina eichhorniae (Coleoptera: Curcu- 
lionidae) en plantas acuáticas de diferente valor nutritivo. Revista Colombiana de Entomología, 39, 81-87.

Thompson, V.\& León, G. R. (2005). La identificación y distribución de los salivazos de la caña de azúcar y los pastos (Homoptera: Cercopidae) en Costa Rica. Manejo Integrado de Plagas y Agroecología, 75, 43-51.

Valdez-Torres, B. J., Soto-Landeros, F., Osuna-Enciso, T. \& BáezSañudo, M. A. (2012). Modelos de predicción fenológica para maíz blanco (Zea mays L.) y gusano cogollero (Spodoptera frugi- perda J.E. Smith). Agrociencia, 46, 399-410.

Valerio, J. \& Koller, W. (1993). Proposição para o manejo integrado das cigarrinhas-das-pastagens. Pasturas Tropicales, 15, 10.

Van Driesche, R. G. \& Bellows, T. S. Jr. (1996). Biological Control. Chapman \& Hall, New York. 539 p.

Velasco-Corona, C., Corona-Vargas, M. C. \& Peña-Martínez, R. (2007). Liometopum apiculatum (Formicidae: Dolichoderinae) y su relación trofobiótica con Hemiptera Sternorrhyncha en Tlaxco, Tlaxcala, México. Acta Zoológica Mexicana (n.s.), 23, 31-42. 